\title{
A SUPERCONDUCTING LANDAU ACCELERATOR MODULE FOR BESSY II
}

\author{
P. vom Stein, M. Pekeler, H. Vogel, ACCEL Instruments, Bergisch Gladbach, Germany \\ W. Anders, BESSY, Berlin, Germany, \\ S. Belomestnykh, J. Knobloch, H. Padamsee, Cornell University, Ithaca, USA
}

\begin{abstract}
The installation of a 3rd harmonic Landau accelerator module is planned at BESSY II to improve the beam lifetime. The passive cavity shall provide an accelerating voltage up to $500 \mathrm{kV}$ for beam currents from 10 to 500 mA. A CESR Phase-III cavity [1], scaled to $1.5 \mathrm{GHz}$, will be used. The cavity design provides strong damping of higher-order modes by ferrite loads on the beam tubes. A new module, constrained to fit into a very limited space at BESSY, is described here. It includes a piezo-electric tuner that allows amplitude control within 1\%. Nonstandard modes of operation, such as normal-conducting stand-by operation and thermal breakdown are also discussed.
\end{abstract}

\section{SYSTEM OVERVIEW}

The Landau accelerator module cavity is based on the CESR superconducting mono mode cavities developed at Cornell University [1]. The cavity design allows the propagation of all HOMs through the beam tubes. On the warm ends of the beam tubes outside the cryostat the HOM power is absorbed in water cooled ferrite loads. The Landau accelerator module will be installed at BESSY II storage ring. The total space for the module is limited to $800 \mathrm{~mm}$ to fit the module into a short straight section. A sketch of the module is shown in figure 1. The basic operating parameters are shown in table 1 .

Table 1: Operating parameters of the Landau module

\begin{tabular}{|l|l|}
\hline frequency & $1.5 \mathrm{GHz}$ \\
\hline $\mathrm{U}_{\text {acc }}(\max )$ & $500 \mathrm{kV}$ \\
\hline $\mathrm{I}_{\text {beam }}(\mathrm{max})$ & $500 \mathrm{~mA}$ \\
\hline r/Q $\left(\mathrm{U}_{\text {acc }}^{2} / 2 \mathrm{P}_{\text {diss }}\right.$ & $45.4 \mathrm{Ohm}$ \\
\hline max. dissipated rf power & $12.5 \mathrm{~W}$ \\
\hline unloaded Q & $2.2 \times 10^{8}$ \\
\hline op. temperature & $4.5 \mathrm{~K}$ \\
\hline standby losses & $<6 \mathrm{~W}$ \\
\hline
\end{tabular}

\subsection{RF couplers and tuning system}

The cavity of the Landau accelerator module runs in passive operating mode. No net acceleration is given to the bunch and no energy transfer also. Depending on the phase difference between the bunch and the rf accelerating voltage the bunch length is increased or decreased. The rf power consumption is caused by the wall losses of the cavity and the rf power extracted by $\mathrm{rf}$ couplers. These losses are compensated by the beam generated rf power. Therefore, only rf couplers with a high external Q are needed. One coupler with an external $\mathrm{Q}$ of approx. $1 \times 10^{8}$ is foreseen for $\mathrm{rf}$ tests and conditioning of the cavity without beam. Two more pick up antennas are installed for rf measurements.

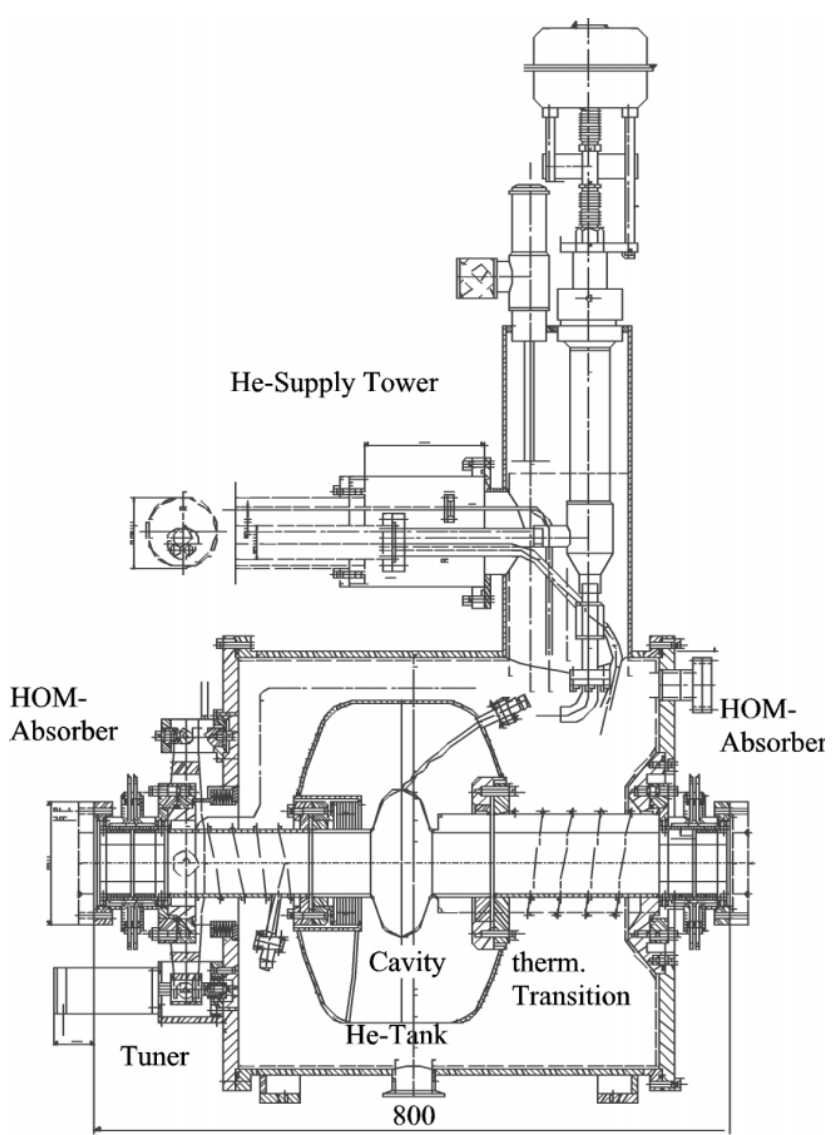

Figure 1: Layout of cryomodule

The cavity frequency has to be tuned precisely next to a harmonic of the bunch revolution frequency of the storage ring. The desired frequency stability of the cavity depends on the beam current, accelerating voltage and amplitude stability [2]. For an amplitude stability of $1 \%$ the required frequency stability is $13 \mathrm{~Hz}$ minimum $\left(\mathrm{I}_{\text {beam }}\right.$ : $\left.10 \mathrm{~mA}, \mathrm{U}_{\text {acc }}: 500 \mathrm{kV}\right)$. This corresponds to a relative positioning accuracy of the tuner of approx. $20 \mathrm{~nm}$. This precision can be achieved by a combined system of a stepper motor for coarse tuning and a piezo translator for fine tuning. The tuner is placed outside the cryostat, which allows an easy maintenance. The tuning forces are transferred by the beam tubes to the cavity. 


\subsection{HOM Loads}

Two water cooled HOM loads are placed on both beam tubes on the outer side of the vacuum vessel. Each load consists of 20 nickel-zinc ferrite tiles brazed to a support structure. The maximum HOM power is approx. $3500 \mathrm{~W}$. This results in a maximum power density on the ferrites of $11.6 \mathrm{~W} / \mathrm{cm}^{2}$. The operating experience at Cornell shows, that these power levels can be handled routinely [3].

\subsection{Cryogenic layout}

The accelerator module is designed for continuous operation at a liquid helium refrigerator. The nominal operating temperature is $4.5 \mathrm{~K}$. The total cryogenic losses are estimated to $20 \mathrm{~W}$. The standby losses are specified to less than $6 \mathrm{~W}$. In case of refrigerator problems the temporary operation of the module with a liquid helium can is foreseen.

One boundary condition for the cryogenic design is the limited length of the module. This results in a short length of the beam tubes between the cavity and the outer cryostat vessel. This thermal transitions are the largest heat leak to the liquid helium temperature level. The beam tubes are equipped with gas counter flow cooling to decrease the heat load to a reasonable level. The gas flow rate through the cooling tubes is controlled by a cold valve integrated in the helium supply tower (see fig. 1).

For the Landau module a radiation shielding by superinsulation was preferred to an active cooled shield. The main reasons are the limited space and the large number of connections between helium vessel and room temperature level. Therefore, the helium vessel and parts of the beam tubes are covered with a relatively large number of 80 layers of superinsulation.

\subsection{Vacuum system}

The beamline vacuum system is designed for UHV $<10^{-8}$ mbar. All flange connections to the isolation vacuum of the cryostat or atmosphere are conflat flanges. This design provides easy installation and a high reliability of the vacuum system. Special copper seals will also provide a good rf contact on the inner side of the beam tube. At the cavity the stainless steel flanges are brazed to the niobium beam tubes. This technique was already proven for several niobium cavities made by ACCEL. The cavity vacuum is pumped by two getter pumps installed in the storage ring vacuum system next to the Landau module.

The requirements for the isolation vacuum are less demanding ( $\mathrm{p}<10^{-5}$ mbar). A small turbomolecular pump provides sufficient pumping speed. The pump will be mechanically decoupled from the cryostat by a bellow. This avoids the transfer of mechanical vibrations to the cavity and the danger of frequency instabilities.

\section{OPERATING MODES}

In normal operating mode the dissipated rf power in the cavity is approximately $12.5 \mathrm{~W}$ at an accelerating voltage of $500 \mathrm{kV}$. For the layout of the safety systems the module performance during system failures is also of interest. The most severe failure will be a cavity quench at maximum beam current $(500 \mathrm{~mA})$ and the cavity tuned next to a revolution frequency harmonic. In this case the cavity will become normal conducting in a time scale of a few milliseconds. On the worst case assumption that the cavity will stay tuned the momentary induced rf losses are in the range of $120 \mathrm{~kW}$. Most probably the beam will be dumped by the power loss. Nevertheless a fast quench detector has to be coupled with the interlock of the main ring rf to assure the shut down of the beam.

The case of stand by operation of the module has also been investigated. This mode might be of special interest to avoid longer shut downs periods in case of severe system failures. For stand by operation the cavity will be in normal conducting state. The frequency has to be tuned in the middle between two revolution frequency harmonics. For moderate beam currents $(100 \mathrm{~mA})$ the induced rf losses will be $150 \mathrm{~W}$. These power levels can be easily led off the cryostat by a warm helium gas circulation system.

\section{REFERENCES}

[1] S. Belomestnykh et al., "Superconducting RF System for CESR Luminosity Upgrade: Design, Status and Plans", Proc. of the $5^{\text {th }}$ EPAC, Sitges (1996), Vol.3, p. 2100-2103

[2] E. Haebel, "Couplers for Cavities", Proc. of the CERN Accelerator School, CERN 96-03, Hamburg (1995), p. 231-264

[3] E. Chojnacki et al., "Beamline RF Load Development at Cornell", Proc. of the 1999 PAC, New York (1999), Vol. 1, p. 845-847 